\begin{tabular}{|c|c|}
\hline \multirow[t]{3}{*}{ Citation } & Xin Wang, Jan Swevers (2013) \\
\hline & $\begin{array}{l}\text { Offset-free Time-optimal Model Predictive Control for Point- } \\
\text { to-Point Motions }\end{array}$ \\
\hline & 32st Benelux Meeting on Systems and Control(2013) \\
\hline Archived version & $\begin{array}{l}\text { Author manuscript: the content is identical to the content of the } \\
\text { published paper, but without the final typesetting by the publisher }\end{array}$ \\
\hline \multicolumn{2}{|l|}{ Published version } \\
\hline \multicolumn{2}{|c|}{ Journal homepage } \\
\hline \multirow[t]{2}{*}{ Author contact } & xin.wang@mech.kuleuven.be \\
\hline & phone number $+32(0) 16322222$ \\
\hline IR & url in Lirias https://lirias.kuleuven.be/handle/123456789/389146 \\
\hline
\end{tabular}




\title{
Offset-free Time-optimal Model Predictive Control for Point-to-Point Motions
}

\author{
Xin Wang ${ }^{1}$, Jan Swevers ${ }^{2}$ \\ Department of Mechanical Engineering, KU Leuven, Celestijnenlaan 300B, B-3001 Heverlee, Belgium \\ Xin.Wang@mech.kuleuven.be, Jan.Swevers@mech.kuleuven.be
}

\section{Introduction}

This presentation discusses time-optimal point-to-point motion control with high positioning accuracy for linear timeinvariant (LTI) systems. An offset-free time-optimal MPC approach is developed based on the time-optimal model predictive control (TOMPC) method and the offset-free MPC method both of which have been proposed in literature [1][2]. In TOMPC, time-optimality is achieved by minimizing the settling time. Offset-free MPC is a disturbance model based MPC approach for accurate positioning.

\section{Background: two existing methods}

Offset-free MPC [1] proposes a general disturbance model that accommodates unmeasured disturbances entering through the process input, state, or output. Given the disturbances estimated by a kalman filter, the target values of the input and state are calculated such that the effect of the disturbances is removed by shifting the input and the state to their target values.

TOMPC [2] is a MPC approach for time-optimal point-topoint motion control of LTI systems. It is formulated as a two layer optimization problem. First the settling time required for the system to be at rest at the desired set point is minimized. Then the optimal signal sequence is obtained by solving the MPC optimization problem. However, TOMPC cannot achieve high positioning accuracy in the presence of unmodelled disturbances or model-plant mismatch. This presentation proposes an extension of TOMPC combining it with offset-free MPC to cope with this problem, and yield so called offset-free time-optimal MPC.

\section{Test setup}

The considered test setup is a current-controlled permanentmagnet linear motor. The control input is motor current $[A]$, and the output is the position of the carriage $[\mathrm{m}]$. The main disturbances in this test setup are Coulomb friction and cogging, which are both modelled as input disturbances and es-

\footnotetext{
${ }^{1}$ Acknowledgement This work has been carried out within the framework of projects IWT-SBO 80032 (LeCoPro) of the Institute for the Promotion of Innovation through Science and Technology in Flanders (IWTVlaanderen). This work also benefits from K.U.Leuven-BOF PFV/10/002 Center-of-Excellence Optimization in Engineering (OPTEC), the Belgian Programme on Interuniversity Attraction Poles, initiated by the Belgian Federal Science Policy Office (DYSCO), and K.U.Leuvens Concerted Research Action GOA/10/11.
}

timated with a Kalman filter. Coulomb friction depends on the sign of the velocity. Cogging is a periodic disturbance function of the position and the period corresponds to the distance between the magnetic poles of the linear motor.

\section{Results and conclusion}

Figure 1 shows some simulation results for a motion step of $0.1 \mathrm{~m}$. TOMPC, offset-free MPC and offset-free TOMPC are compared. The input currents are within the constraints $\pm 3 A$. It is obvious to see that the offset-free TOMPC achieves the best performance (shortest settling time and smallest steady-state error). The results are also summarized in the following table. Experimental validation on the linear motor setup is in progress.

(a)

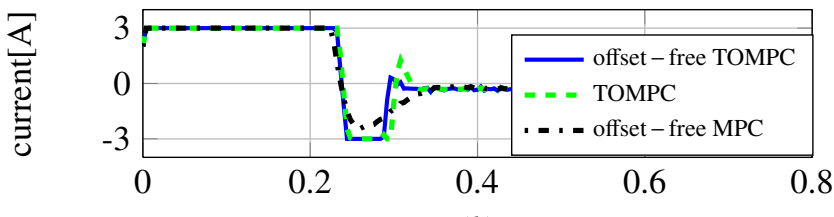

(b)

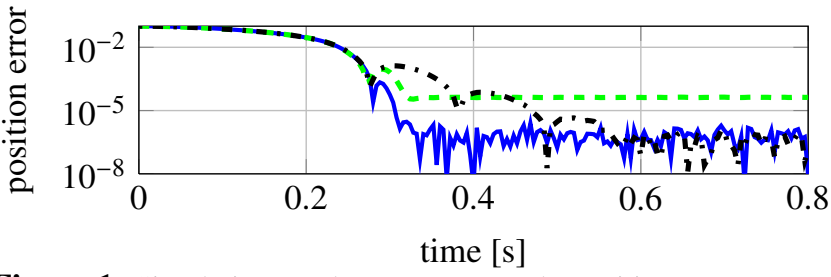

Figure 1: Simulation results: (a) currents (b) position errors

\begin{tabular}{|c|c|c|}
\hline Methods & settling time & steady-state error \\
\hline offset-free MPC & $0.58[\mathrm{~s}]$ & $5 \times 10^{-7}[\mathrm{~m}]$ \\
\hline TOMPC & $0.32[\mathrm{~s}]$ & $1 \times 10^{-4}[\mathrm{~m}]$ \\
\hline offset-free TOMPC & $0.32[\mathrm{~s}]$ & $5 \times 10^{-7}[\mathrm{~m}]$ \\
\hline
\end{tabular}

\section{References}

[1] G. Pannocchia1, J.B. Rawlings, Disturbance models for offset-free model-predictive control, vol. 49, no. 2, AIChE Journal, 2003, pp. 426-437.

[2] Van den Broeck,L., Diehl,M., Swevers,J., A model predictive control approach for time optimal point-to-point motion control, vol. 21, no. 7, Mechatronics, 2011, pp. 1203-1212. 